\title{
Wärmetransportoptimierung in Biogasanlagen durch innovative Wärmeübertrager und Konzeptevaluation mittels CFD-Methoden
}

\author{
B.Sc. Chris Geiger, Technische Universität München, Freising
}

\begin{abstract}
Kurzfassung
Durch die Novellierung des EEG 2017 steigen die Anforderungen an die Energieertragseffizienz von Biogasanlagen. Die bei herkömmlichen Anlagen an der Fermenterwand platzierten Heizsysteme sind für die Energieübertragung suboptimal. Zur Steigerung des Wirkungsgrades von Biogasanlagen werden neuartige Konzepte aufgezeigt, welche die Vorteile der Wärmeübertragung durch Zwangskonvektion in Gebieten hoher Strömungsgeschwindigkeiten optimal nutzen. Zeitgleich werden die Wandwärmeverluste durch eine Verlagerung der Bereiche mit höchster Fluidtemperatur in das Fermenterzentrum reduziert. Hierfür sieht ein Konzept die Koppelung eines Rohrwärmeübertragers mit einem Propellerrührwerk vor. Des Weiteren wird ein Konzept zur Funktionskombination der Homogenisierung und des Wärmeeintrags mittels der Nutzung von Axialrührwerken als Mischeinrichtung und Wärmetauscher untersucht. Der Einfluss der Paddelgeometrie und des Anstellwinkels auf eine optimale Durchmischung und Wärmeeintrag bei variierender Drehzahl steht im Fokus der Untersuchungen. Die mit numerischen Strömungssimulationen evaluierten Konzepte sind für eine effiziente Abdeckung des benötigten Wärmebedarfs von Biogasanlagen bei guter Durchmischung sehr geeignet.
\end{abstract}

\section{Einführung}

Das Ziel der zukünftigen Energieproduktion ist es, durch einen großen Anteil an regenerativen Energien am Energiemix eine Minimierung der anthropogenen KohlenstoffdioxidEmission zu erreichen. Für die Förderung erneuerbarer Energien zur Emissionsminimierung trat bereits im Jahr 2000 das ersten Erneuerbare-Energien-Gesetz (EEG) in Kraft [1]. Die Novellierung des EEG 2017 fordert eine Steigerung der Effizienz und Flexibilität von Biogasanlagen für eine ökonomische Arbeitsweise ohne gesetzlich festgelegte Vergütungen des produzierten Stromes [2]. Ziel dieser Studie ist die Entwicklung und Evaluation von Konzepten zur Optimierung des Wärmeeintrags und der Homogensierung des Gärsubstrates in Biogasanlagen auf Basis aktueller Anlagenkomponenten, welche in einer Effizienzsteigerung resultiert. 


\section{Grundkonzepte zur Effizienzsteigerung}

Eine Verringerung der Wandwärmeverluste des Fermenters bietet eine gute Basis zur Effizienzsteigerung von Biogasanlagen. Diese sind nach [3]:

$$
\dot{Q}_{\text {Verlust }}=U \mathrm{~A} \cdot\left(T_{\text {Fluid }}-T_{\infty}\right)
$$

In herkömmlichen Anlagen sind die Heizrohre direkt an oder in der Wand verbaut, wodurch die Maximaltemperatur direkt an dieser anliegt und der Verlustwärmestrom maximal ist [1]. Dieser kann ohne Veränderungen der Bauform oder des Volumens des Gärbehälters allein durch eine Reduktion der Temperaturdifferenz minimiert werden. Hierfür erfolgt die Wärmezufuhr im Fermenter wandfern, optimaler Weise in der Behältermitte. Durch dieses Grundkonzept, dargestellt in Bild 1, ist die Temperatur des Gärsubstrates in Wandnähe minimal, womit nach Gleichung (1) die Wärmeverluste reduziert werden.

Der spezifische Wärmeeintrag in das Gärsubstrat durch den

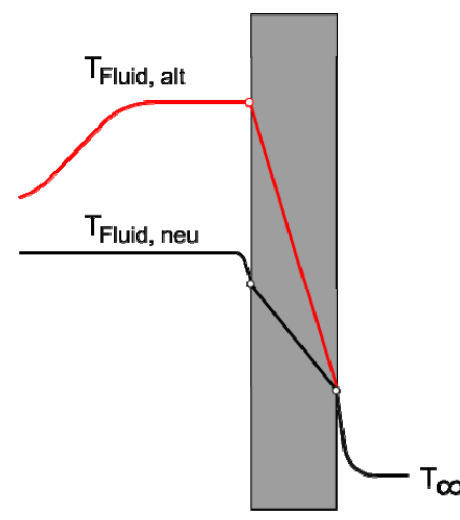

Bild 1:

Grundidee zur Optimierung Wärmeübertrager wird mittels der Wärmestromdichte durch Gleichung (2) allgemein beschrieben [3]:

$$
\dot{q}_{\text {Heizelement }}=\alpha \cdot\left(T_{\text {Heizelement }}-T_{\text {Fluid }}\right)
$$

Die Erhöhung des Wärmeübertrags erfolgt durch eine Steigerung von a mittels Erhöhung der erzwungenen Konvektion. Dies geschieht durch einen Anstieg der Fluidgeschwindigkeit an der wärmeübertragenden Fläche, wodurch bei gleicher Wärmestromdichte die Temperaturdifferenz gesenkt werden kann. Die geringere lokale Überhitzung des Fluides bei gleichzeitig geringerem Bauraum des Wärmeübertragers ist vorteilhaft.

\section{Konzeptvarianten}

Die in Kapitel 0 ausgeführten Ansätze sind als zwei unterschiedliche Konzepte mit mehreren Varianten realisiert. Zum einen ist ein Konzept für bestehende Fermenter mit Propellerrührwerken dargestellt. Ein als durchströmtes Rohr ausgeführter Wärmetauscher, siehe Bild 2, wird vom Gärsubstrat zwangsdurchströmt. Der Wärmeeintrag findet über die Rohrwand statt. Alternativ wird ein Konzept vorgestellt, welches bei einem Neubau einer Anlage Anwendung finden soll. Das Axialrührwerk fungiert simultan als Wärmetauscher und Rührwerk, wodurch der Wärmeeintrag und die Homogenisierung in einer Anlagenkomponente kombiniert wird. Nachfolgend sind die effizientesten Konfigurationen der jeweiligen Konzepte detailliert erläutert. 
Wellrohr: Das wellenförmige Profil, dargestellt in Bild 2, weist einen Durchmesser von $D=1$ $\mathrm{m}$ und einer Länge von $\mathrm{L}=1 \mathrm{~m}$ auf und ist von Produkten aus dem Baugewerbe abgeleitet. Die Auswahl des Durchmessers erfolgt unter Berücksichtigung des Propellerdurchmessers eines repräsentativen Rührwerks von 900 mm, die Definition der Rohrlänge fand mit dem Augenmerk auf eine möglichst kompakte Bauform statt. Mit dem doppelwandigen Aufbau sind mehrere Heizkreise möglich, wodurch eine konstante Wandtemperatur sichergestellt wird. Vorteil dieses Konzepts ist eine Vermeidung der Kavernenbildung um das Propellerrührwerk und eine Förderung des erwärmten Fluides zur Fermentermitte hin [4]. Das Wärmetauscherkonzept wird numerisch als Kreissegment hinsichtlich des zu erwartenden Strömungsregimes sowie des Wärmeübergangs untersucht.

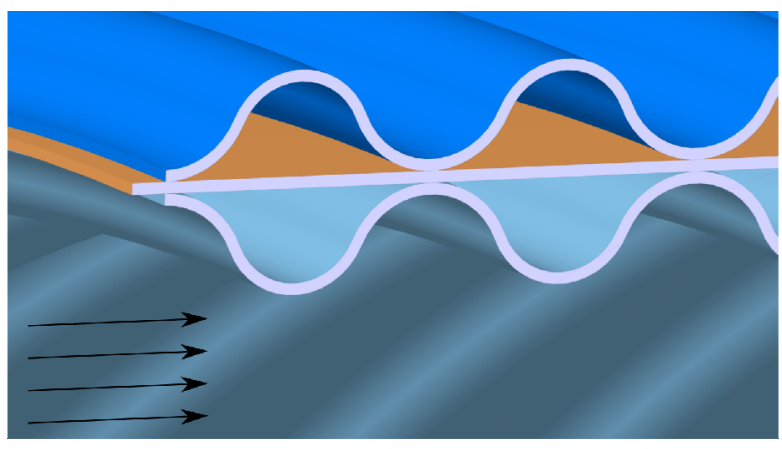

Bild 2: Schnittbild des Wellrohr-

Paddelrührwerk: Bei diesem Konzept wird Wärmeübertrager der gesamte Fermenter betrachtet, in welchem sich das Rührwerk befindet. Die Maße des Fermenters entsprechen mit ca. $883 \mathrm{~m}^{3}$ und einem Rührwerksdurchmesser von $\mathrm{D}=3.2 \mathrm{~m}$ der Bauform einer kleinen Biogasanlage. Bei der Gittergenerierung wurde, wie auch bei dem Konzept des Wellrohres, auf eine Verfeinerung des Gitters in Wandnähe des Wärmeübertragers mit $\mathrm{y}^{+}<1$ geachtet, um den Wärmeeintrag optimal abzubilden. Eine Variation der Paddelgeometrie ergibt die in Bild 3 dargestellte Geometrie eines angepassten NACA 4404Profils sowie die der Form A und B. Für den Entwurf der Form A sind die Ergebnisse einer Studie zu optimierten Paddelprofilen berücksichtigt [5]. Jeweils für die Paddelformen A und B wird das Strömungsverhalten und der Wärmeübergang bei einem Anstellwinkel (PA) von $15^{\circ}, 30^{\circ}, 45^{\circ}$ und $90^{\circ}$ simuliert. Das NACA 4404-Profil, welches besonders für geringe Reynoldszahlen geeignet ist, wird in seinem optimalen Anstellwinkel von $10^{\circ}$ betrachtet [7]. Je Anstellwinkel findet eine Variation der Drehzahl des Rührwerkes statt.

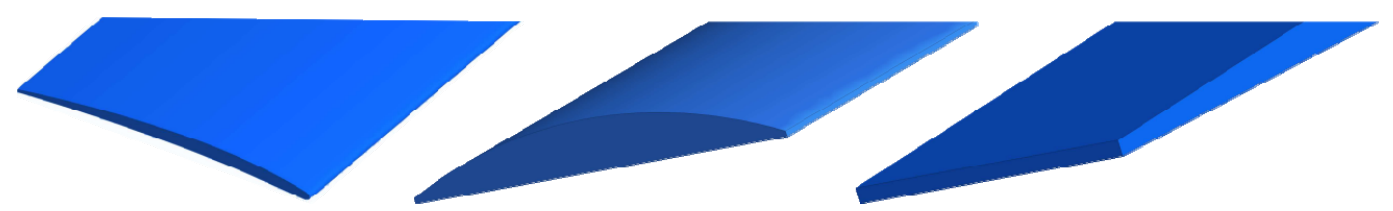

Bild 3: Profilformen: Links NACA 4404, Mitte Form A, Rechts Form B

Randbedingungen: Der reibungsbehafteten Wand der Wärmetauscher wird eine Temperatur von $323.15 \mathrm{~K}$ aufgeprägt, dem Fluid von $313.15 \mathrm{~K}$, was der Substrattemperatur einer mesophil betriebenen Anlage entspricht [6]. Die Temperaturdifferenz ist für eine möglichst ge- 
ringe Vorlauftemperatur des Heizkreises und den damit verbundenen energetische Aufwand zu 10 K gewählt. Am Einlass des Wellrohres wird eine Anströmgeschwindigkeit vorgegeben, welche je Simulation in einem Bereich von $1 \mathrm{~m} / \mathrm{s}$ bis zu $5 \mathrm{~m} / \mathrm{s}$ variiert. Für das Paddelrührwerk werden Drehzahlen von $5 \mathrm{U} / \mathrm{min}, 10 \mathrm{U} / \mathrm{min}$ und $15 \mathrm{U} / \mathrm{min}$ betrachtet. Das nichtnewtonsche Verhalten des Fluides ist über den Ostwald und de Waele - Ansatz modelliert [8]

\section{Auswertung der numerischen Untersuchungen}

Wellrohr: Der Wärmestrom ist in Bild 4 über die Anströmgeschwindigkeit aufgetragen. Er steigt dabei annähernd quadratisch an. Dies begründet sich durch das nicht-newtonschen Verhalten des Fluides in Kombination mit dem wellenförmigen Profil der Rohrwand. Durch die Steigerung der Anströmgeschwindigkeit erfährt das Fluid an der wellenförmigen Oberfläche eine deutlich größere Scherung. Das pseudo-plastische Verhalten führt zu einer Reduktion der Viskosität. Zeitgleich ist die Wirbelstärke



Bild 4: Wärmestrom des WellrohrKonzeptes

der Wirbelstrukturen in der Wellung größer. Dieser Effekt führt mit dem lokal niedrigviskoserem Fluid zu einer erhöhten Geschwindigkeit im Bereich der Wellung und damit zu einem maximierten Wärmestrom.

Paddelrührwerk: Für einen Vergleich der verschiedenen Rührwerksvarianten als Mischeinrichtung und Wärmetauscher ist in Bild 5 der Wärmestrom über die Leistung aufgetragen. Für eine übersichtliche Darstellung wird nur der Leistungsbereich bis $3.5 \mathrm{~kW}$ betrachtet. Betriebspunkten mit höherer Leistung sind bedingt durch den vergleichbar großen relativen Leistungseintrag praktisch irrelevant. Bei $15 \mathrm{U} / \mathrm{min}$ ist der Wärmestrom für jede Variante maximal. Mit einer Betrachtung des Wärmestroms bei gleicher Leistung lässt sich die Effizienz der Rührwerke untersuchen. In einem

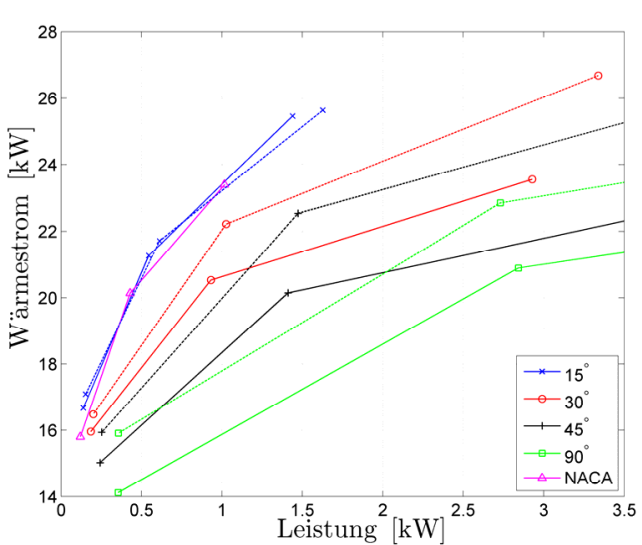

Bild 5: Vergleich des Wärmestroms für die Konfiguration der Axialrührwerke; ( - ) Form A, ( - - ) Form B Leistungsbereich um $1.5 \mathrm{~kW}$ weisen diese bauformunabhängig bei einem Anstellwinkel von $P A=15^{\circ}$ den höchsten Wärmestrom auf. Allen Profilen ist ein sehr ähnlicher Verlauf der 
Wärmestrom-Leistungskurve zu Eigen. Eine Erhöhung des Anstellwinkels führt zu einem gesteigerten Leistungsbedarf der Rührwerke, die Unterschiede zwischen den Paddelformen steigen ebenfalls an. So weist bei PA $=30^{\circ}$ die Bauform $B$ ein um $13.3 \%$ erhöhten Wärmestrom auf, bei einer Leistungssteigerung um 14\%. Der erhöhte Wärmestrom der Form B ist auf eine stärkere lokale Bildung von Wirbelstrukturen an den Profilkanten und auf die im Vergleich zur Form A um 11.5\% größer Oberfläche zurückzuführen. In Hinblick auf die Wärmestromdichte ergibt sich jedoch eine höhere Effizienz für die Bauform A und des NACA 4404-Profils aufgrund der geringeren Rührwerksfläche bei annähernd gleichem Wärmestrom. Bei der Profilform A tritt ab PA $=30^{\circ}$ eine Ablösung der Strömung an der Paddeloberfläche auf, wodurch der Wärmestrom abnimmt. Erst bei einer Drehzahl von $15 \mathrm{U} / \mathrm{min}$ und $\mathrm{PA}=90^{\circ}$ übertrifft der Wärmestrom die Ausgangsvariante $\left(15 \mathrm{U} / \mathrm{min}, \mathrm{PA}=15^{\circ}\right)$. Der Grund hierfür ist das Rezirkulationsgebiet an der Paddelrückseite mit einer starken Wirbelbildung und hohen Fluidgeschwindigkeiten. Die Wärmestromdichte und die Stromlinien stimmen hierbei mit einer Studie über angeströmte Halbkreis-Profile überein [9]. Bild 6 zeigt die Geschwindigkeitsverteilung und Stromlinien des Gärsubstrates bei $15 \mathrm{U} / \mathrm{min}$ und der Paddelform A. Im Bereich des Rührwerkes bildet sich eine globale Wirbelstruktur und in Fermenterwandnähe zwei großskalige, gegenläufige Wirbelgebiete. Die Drehrichtung des oberen Wirbels rechts der Rührachse ist im Uhrzeigersinn, die des unteren entgegengesetzt. Damit wird tendenziell kälteres Fluid aus dem Wandbereich zum Rührwerk hin gefördert. Die Durchmischung ist mit der in [10] beschrieben Methode untersucht worden. Das Gärsubstrat ist bei allen Varianten vollständig homogenisiert.
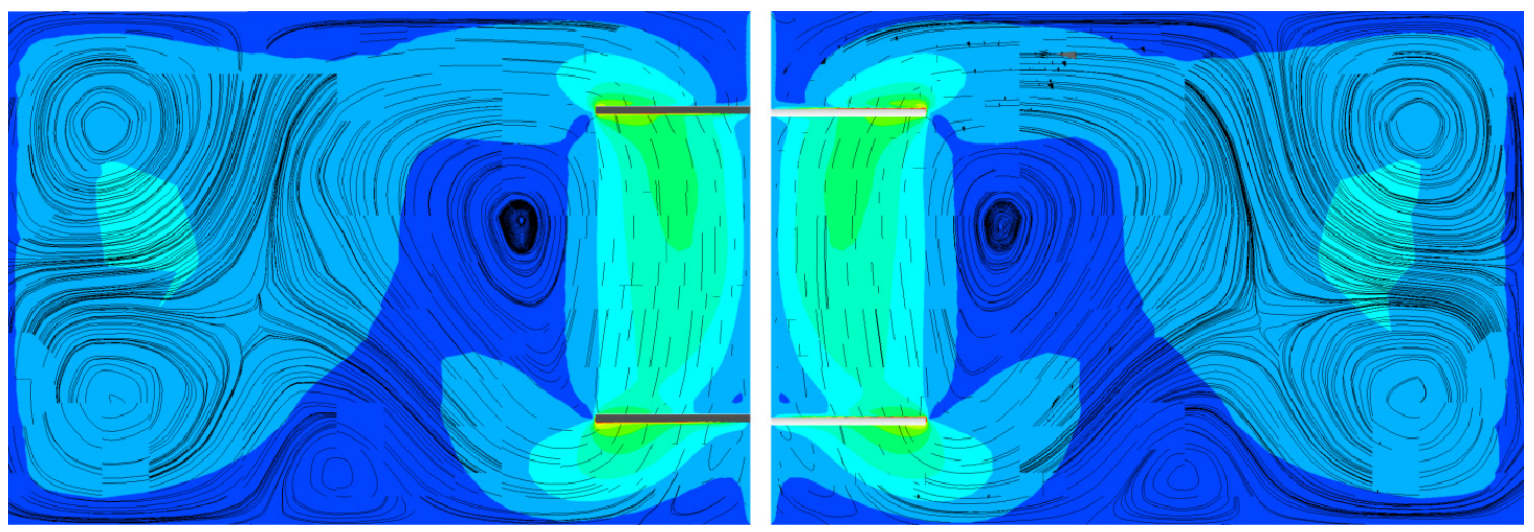

0

0.5

1

Geschwindigkeit $[\mathrm{m} / \mathrm{s}]$

Bild 6: Globale Geschwindigkeit und Stromlinien bei $15 \mathrm{U} / \mathrm{min}, \mathrm{PA}=15^{\circ}$, Form A 


\section{Diskussion und Evaluation der Ergebnisse}

Mit den dargestellten Konzepten ist eine Abdeckung des Wärmebedarfes in Biogasanlagen möglich. Durch die große Varianz dessen, resultierend aus unterschiedlicher Baugröße und Isolierung, ist eine spezifische Anpassung des Wärmeübertragers auf die jeweilige Anlage zu empfehlen. Zusätzlich zu der Grundversorgung der Anlagen ist eine temporäre Steigerung des Wärmestromes durch eine erhöhte Wandtemperatur zum Zeitpunkt der Fütterung möglich. Pohn [10] empfiehlt die Positionierung eines Propellerrührwerks nahe der Einbringstelle, um eine optimale Verteilung der Frischmasse zu erreichen. Diese weist eine geringere Temperatur als das Gärsubstrat auf. Mit einer kurzzeitigen Temperaturerhöhung des Wärmetauschers kann die Frischmasse schneller auf die Fluidtemperatur erwärmt werden, da diese direkt nach Einbringung in den Fermenter durch das beheizte Wellrohr gefördert wird. Dies führt zu einer homogeneren Temperaturverteilung innerhalb des Gärsubstrates. Bei den Axialrührwerken besteht ebenfalls die Möglichkeit einer temporären Erhöhung der Temperatur. Werden die Paddelflächen beispielsweise elektrisch beheizt, ist dies sogar mit einer sehr geringen Vorlaufzeit möglich. Dadurch können Temperaturschwankungen, wie bei einem Eintrag von Frischmasse mit variierender Menge und Temperatur, sehr gut reguliert werden. Die Tabelle 1 stellt zusammenfassend je Konfiguration die Wärmemenge dar, welche in das Gärsubstrat eingebracht werden kann.

Tabelle 1: Vergleich ausgewählter Konzepte mit optimaler Effizienz

\begin{tabular}{ccc}
\hline \hline Bauform & Rührzeit $[\mathrm{h} / \mathrm{d}]$ & Eingebrachte Wärmemenge $[\mathrm{kWh} / \mathrm{d}]$ \\
\hline Wellrohr & 4.8 & 499.2 \\
Paddelform A & 24 & 611.0 \\
Paddelform B & 24 & 639.1 \\
NACA-4404 & 24 & 561.4 \\
\hline
\end{tabular}




\section{Literatur}

[1] Döhler, H.: Faustzahlen Biogas. Darmstadt: KTBL 2009.

[2] Bundesministerium für Wirtschaft und Energie: EEG - Novelle 2017: Kernpunkte des Bundestagsbeschlusses vom 8.7.2016

[3] Polifke, W., Kopitz, J.: Wärmeübertragung: Grundlagen, analytische und numerische Methoden. München: Pearson 2009.

[4] Rostalski, K.:Rührwerke in Biogasanlagen: Technik mit zentraler Bedeutung. Biogas in der Landwirtschaft - Stand und Perspektiven. KTBL/FNR-Biogas-Kongress (2009) S. 26-36

[5] Casartelli, E., Waser, R., Frankhauser, H.: Strömungstechnische Optimierung eines Biomasse-Rührwerks. Schlussbericht 2013.

[6] Kaltschmitt, M., Hartmann, H., Hofbauer, H.: Energie aus Biomasse. Berlin: SpringerVerlag 2009

[7] Kunz, P.J.:AERODYNAMICS AND DESIGN FOR ULTRA-LOW REYNOLDS NUMBER FLIGHT., Stanford University Diss. 2003.

[8] Brehmer, M., Eppinger, T., Kraume, M.: Einfluss der Rheologie auf das Strömungsregime in gerührten großtechnischen Biogasreaktoren. Chemie Ingenieur Technik 84 (2012) 11 S. 2048-2056.

[9] Tiwari, A.K., Chhabra, R.P.:Momentum and Heat Transfer Characteristics for the Flow of Power-Law Fluids over a Semicircular Cylinder. Numerical Heat Transfer, Part A 66 (2014) 12 S. 1365-1388.

[10] Pohn, S.: Minimierung des Energieverbrauchs von Rührwerken in Biogasfermentern mittels CFD Methoden., Technische Universität Wien Diss. 2012. 
